\title{
TOPOLOGY OPTIMIZATION OF COMPLIANT MECHANISM DESIGN WITH STA- TIONARY FLUID-STRUCTURE INTERACTION
}

\author{
G. H. Yoon ${ }^{1}$ \\ ${ }^{1}$ Assistant Professor, School of Mechanical Engineering, Hanyang University, Republic of \\ Korea (ghy@hanyang.ac.kr/gilho.yoon@gmail.com)
}

\begin{abstract}
This paper outlines a new procedure for topology optimization in the steady-state fluid-structure interaction (FSI) problem. A review of current topology optimization methods highlights the difficulties in alternating between the two distinct sets of governing equations for fluid and structure dynamics (hereafter, the fluid and structural equations, respectively) and in imposing coupling boundary conditions between the separated fluid and solid domains. To overcome these difficulties, we propose an alternative monolithic procedure employing a unified domain rather than separated domains, which is not computationally efficient. In the proposed analysis procedure, the spatial differential operator of the fluid and structural equations for a deformed configuration is transformed into that for an undeformed configuration with the help of the deformation gradient tensor. For the coupling boundary conditions, the divergence of the pressure and the Darcy damping force are inserted to the solid and fluid equations, respectively. The proposed method is validated in several benchmark analysis problems. Topology optimization in the FSI problem is then made possible by interpolating Young's modulus, the fluid pressure of the modified solid equation, and the inverse permeability from the damping force with respect to the design variables.
\end{abstract}

Keywords: Topology optimization,

topology optimization, fluid-structure interaction, monolithic approach

\section{INTRODUCTION}

The numerical simulation and the optimization of fluid-structure interactions have been important subjects in engineering [1,2,3,4,5,6]. Many innovative numerical analysis procedures have been developed which can be mainly categorized into staggered (partitioned) and monolithic analysis procedures depending on the differences in the coupling method. In staggered analysis procedures, the fluid and the structure equations are alternately solved in time, and the interface coupling boundary conditions in traction and velocity are enforced asynchronously. On the other hand, in monolithic analysis procedures, the coupling interaction along the interfacing boundary between the fluid and the structure is treated synchronously, as 
shown in Figure 1. Depending on the problem characteristics of FSI systems, either of the procedures can be employed $[2,3,4,5,6]$.

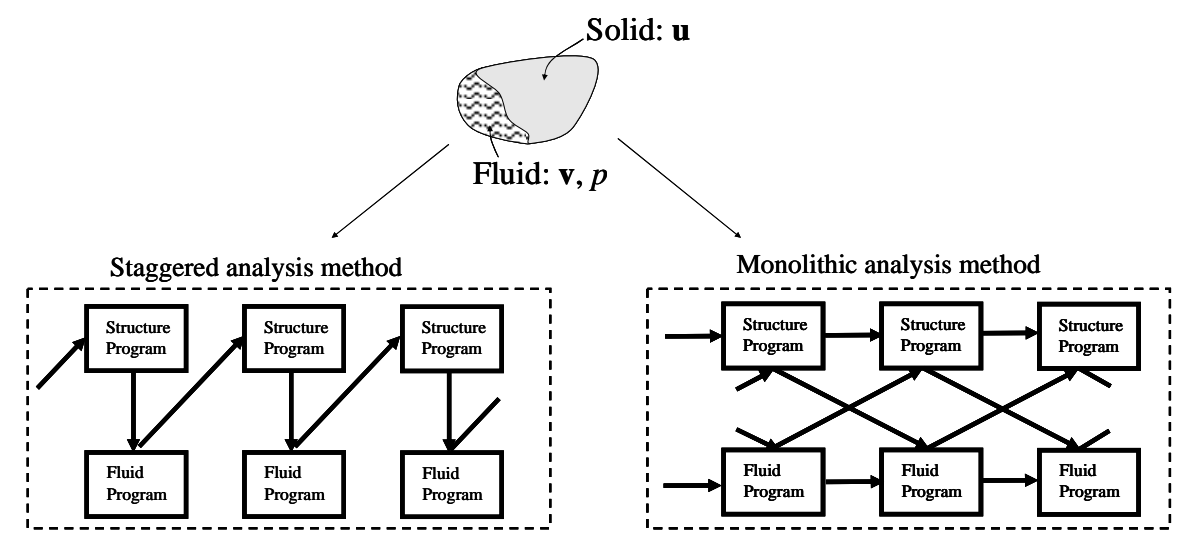

Figure 1. Staggered and monolithic analysis method with separate analysis domains [4].

One of the motivations of the present research is that when applying the existing staggered or monolithic numerical schemes for FSI to one of the structural optimization schemes called topology optimization many complications exist: alternating the distinct governing equations with respect to the density design variable and imposing the explicit coupling boundary condition. Thus, in this research, we employ a new FE-based monolithic procedure with a unified analysis domain for solving steady-state fluid-structure interaction problems and apply this monolithic analysis procedure to topology optimization of compliant mechanism design considering FSI $[2,3,4]$. One of the main differences between the present monolithic approach and previous staggered and monolithic procedures lies in that fact that a unified analysis domain having both the linear elasticity and the Navier-Stokes equation is employed with the coupling boundary conditions. In this new monolithic approach, it is possible to alternatively interpolate the two governing equations by interpolating the material properties involved with the two equations. In the present research, we apply our previous analysis formulation and investigate the disadvantages and advantages of the developed analysis procedure in more detail. 


\section{UNIFIED FE FORMULATION FOR STEADY STATE FLUID STRUCTURE IN- TERACTION}

Because the present study focuses on the reformulation of the governing equations as well as the coupling boundary condition using the involved material properties of the Navier-Stokes equation and the linear elasticity equation without explicit interaction boundary curves, only the steady-state FSI problem is considered. Furthermore, only small structural displacements are assumed for the structural engineering strain, i.e., linear strain, which make the stiffness matrix independent of the structural displacements. The fluid induced force in the linear elasticity equation is dependent on the structural displacements. For more deail descriptions, see our contribution in $[2,3,4]$.

To define the unified formulations for structure and fluid, we introduced the transformation of the differential operators, $\mathbf{F}$. The finite deformation tensor, $\mathbf{F}$, is defined as the partial differential of the current coordinate, $\mathbf{x}$, with respect to the undeformed coordinate, $\mathbf{X}$.

$$
\begin{gathered}
\nabla_{\mathbf{x}} \mathbf{u}=\mathbf{F}^{-\mathrm{T}} \nabla_{\mathbf{X}} \mathbf{u}, \nabla_{\mathbf{x}} \mathbf{v}=\mathbf{F}^{-\mathrm{T}} \nabla_{\mathbf{X}} \mathbf{v} \text {, and } \int_{{ }^{t} \Omega}() d \Omega=\int_{0_{\Omega}}()\|\mathbf{F}\| d \Omega \\
-\int_{0_{\Omega}} \delta \mathbf{v}^{\mathrm{T}}\left\{\rho\left(\mathbf{v} \cdot \mathbf{F}^{-\mathrm{T}} \nabla_{\mathbf{X}} \mathbf{v}\right)\right\}\|\mathbf{F}\| d \Omega=\int_{0_{\Omega}} \mathbf{F}^{-\mathrm{T}} \nabla_{\mathbf{X}} \delta \mathbf{v}^{\mathrm{T}} \mathbf{T}_{f}\|\mathbf{F}\| d \Omega+\int_{0_{\Omega}} \alpha \delta \mathbf{v}^{\mathrm{T}} \mathbf{v}\|\mathbf{F}\| d \Omega-\int_{{ }_{0} \Gamma_{p^{*}}} p_{p^{*}} \mathbf{n} d \Gamma \\
-\int_{0_{\Omega}} \delta p^{\mathrm{T}}\left\{\left(\nabla_{\mathbf{x}} \cdot \mathbf{v}\right)\right\}\|\mathbf{F}\| d \Omega=0 \\
\int_{{ }_{0} \Omega} \delta \mathbf{S}^{\mathrm{T}} \cdot \mathbf{T}_{s} d \Omega=\int_{{ }_{0} \Omega} \Psi \cdot \mathbf{F}^{-\mathrm{T}} \delta \mathbf{S}(\mathbf{u}, \delta \mathbf{u})^{\mathrm{T}} \cdot p\|\mathbf{F}\| d \Omega+\int_{{ }_{0} \Omega} \Psi \cdot \mathbf{F}^{-\mathrm{T}} \delta \mathbf{u} \cdot \nabla_{\mathbf{x}} p\|\mathbf{F}\| d \Omega
\end{gathered}
$$

where the fluid velocity field and pressure of an incompressible flow are described by $\mathbf{v}$ and $\mathrm{p}$, respectively. Note that the differential operator, $\nabla_{\mathbf{x}}$, at time $t$ is defined at the control volume, ${ }_{f}^{t} \Omega(\mathbf{u})$, which implies that the control volume is dependent on the structural displacements, $\mathbf{u}$. The dynamic viscosity is $\mu$ for the Newtonian flow. The Dirichlet boundary conditions are imposed on ${ }_{f}^{t} \Gamma_{\mathbf{v}^{0}},{ }_{f}^{t} \Gamma_{\mathbf{v}^{*}}$, and ${ }_{f}^{t} \Gamma_{i}(\mathbf{u})$ for the no-slip boundary condition, inflow/outflow boundary condition, and interfacing boundary condition, respectively. The Neumann boundary condition for the applied pressure, $p_{p^{*}}$, is defined at ${ }_{f}^{t} \Gamma_{p^{*}}(\mathbf{u})$ with the normal vector, $\mathbf{n}$. The linear strain $\mathbf{S}$ and associate stress $\mathbf{T}_{s}$ are defined. 


\section{TOPOLOGY OPTIMIZATION EXAMPLE}

The syntheses of the compliant mechanism layouts maximizing the output displacements at the different locations of the springs in Figure 2 are considered using the present monolithic analysis scheme. The objective of the topology optimization is to distribute an allowed mass at the design domain (the right domain) in order to maximize the structural $\mathrm{x}$-displacement at the spring simulating a workpiece as (5).

$$
\begin{aligned}
& \operatorname{Max} \Phi=u_{s} \\
& \text { Subject to } \sum_{e=1}^{N E D} \gamma_{e} \mathrm{v}_{e} \leq V^{*}
\end{aligned}
$$

where the objective function , $\Phi$, is the displacement for the spring $\left(u_{s}\right)$. The element volume and the upper bound of the volume are denoted by $\mathrm{v}_{e}$ and $V^{*}$, respectively. The volume limit is constrained to be less than $10 \%$ of the design domain.

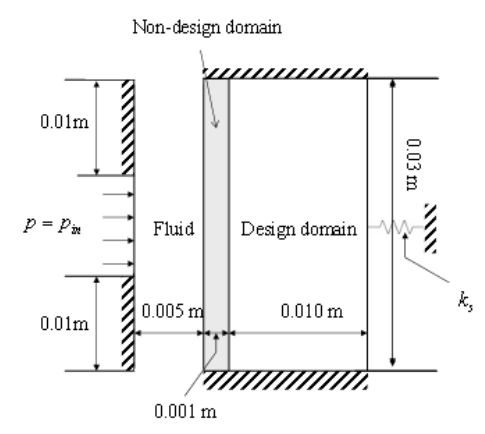

Compliant mechanism 1

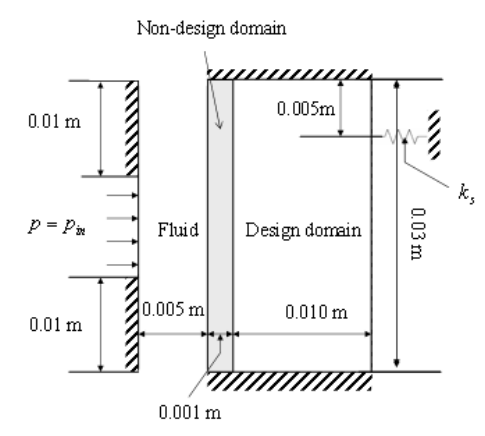

Compliant mechanism 2

(a)
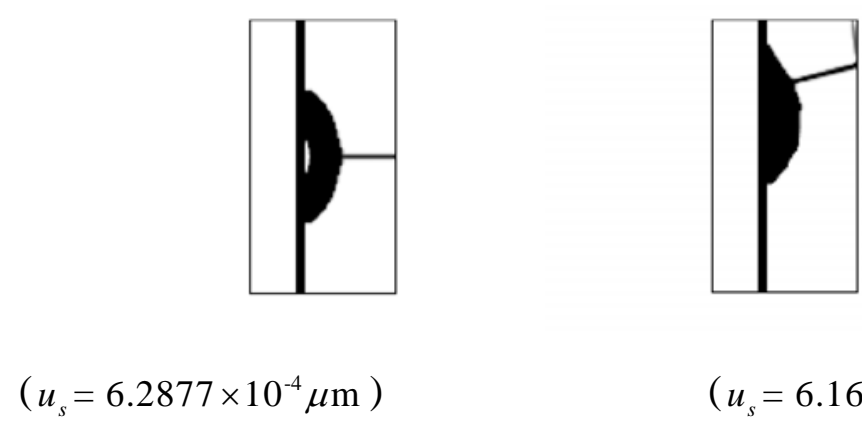

$$
\left(u_{s}=6.1682 \times 10^{-4} \mu \mathrm{m}\right)
$$

(b)

Figure 2. Problem definition of the compliant mechanisms and the obtained layouts [4]. ( $\rho=$ $1000 \mathrm{~kg} / \mathrm{m}^{3}, \mu=1.002 \mathrm{mkg} / \mathrm{ms}, C_{s}=0.1 \mathrm{MPa}, v=0.3, V^{*}: 15 \%, \alpha_{\max }=10^{12}, p_{\text {in }}=0.01$ $\left.\mathrm{N} / \mathrm{m}^{2}, k_{s}=1000 \mathrm{~N} / \mathrm{m}\right)$ 
Using a uniform initial design $\left(\gamma_{\text {initial }}=0.15\right)$ satisfying the mass constraint, the optimal layouts can be obtained as Figure 2(b) for the different spring locations. It is likely that the layouts transmitting the fluid force to the springs are obtained.

\section{CONCLUSIONS}

In order to obtain optimal compliant mechanisms, this paper develops a monolithic formulation based on our previous formulation for FSI analysis. Common staggering or monolithic analysis methods have been used in the analysis and size/shape optimization of FSI systems, but the analysis procedures become prohibitively complicated in the case of density-based topological optimization. To resolve this issue, our previous analysis formulation and procedure have been implemented and applied; the stress predictions are improved. We solve topology optimization for the compliant mechanism considering fluidstructure interaction to show the validity of the developed approach in optimization. The detail formulations and examples will be reported in [4].

\section{REFERENCES}

[1] Bendsøe M. P., Sigmund O., “Topology Optimization Theory Methods and Application”. Springer-Verlag. 2003.

[2] Yoon G.H., "Topology optimization for stationary fluid-structure interaction problems using a new monolithic formulation”. International Journal for Numerical Methods in Engineering , 82,591-616, 2010.

[3] Yoon G. H., “Topological layout design of electro-thermal-compliant actuator.” Computer Methods in Applied Mechanics and Engineering ,209-212,28-44, 2012.

[4] Yoon G. H., "Monolithic fluid-structure interaction analysis for topological compliant mechanism design", in review.

[5] Andreasen C., Sigmund O., "Saturated poroelastic actuators generated by topology optimization.” Structural and Multidisciplinary Optimization 43,693-706, 2011.

[6] Kreissl S., Pingen G., Evgrafov A., Maute K., "Topology Optimization of Flexible Micro-Fluidic Devices.” Structural and Multidisciplinary Optimization, 42(4):495-516, 2010. 